\title{
A Qualitative Study of Barriers to Effectiveness of Interventions to Prevent Mother-to-Child Transmission of HIV in Arba Minch, Ethiopia
}

\author{
Adebola Adedimeji, ${ }^{1}$ Nareen Abboud, ${ }^{1}$ Behailu Merdekios, ${ }^{2}$ and Miriam Shiferaw ${ }^{1}$ \\ ${ }^{1}$ Centre for Public Health Sciences, Albert Einstein College of Medicine, 1300 Morris Park Avenue, Bronx, New York, NY 10461, USA \\ ${ }^{2}$ College of Medicine and Health Sciences, Arba Minch University, Arba Minch, Ethiopia
}

Correspondence should be addressed to Adebola Adedimeji, adebola.adedimeji@einstein.yu.edu

Received 30 March 2012; Revised 23 May 2012; Accepted 11 June 2012

Academic Editor: Pranitha Maharaj

Copyright ( $\odot 2012$ Adebola Adedimeji et al. This is an open access article distributed under the Creative Commons Attribution License, which permits unrestricted use, distribution, and reproduction in any medium, provided the original work is properly cited.

\begin{abstract}
Objectives. Despite the availability of services to prevent mother-to-child transmission (PMTCT) of HIV, socio-cultural, health system and operational factors constrain many pregnant women from accessing services or returning for followup thereby increasing the risk of vertical transmission of HIV to newborns. We highlight and describe unique contextual factors contributing to low utilization of PMTCT services in Arba-Minch, Ethiopia. Methods. Qualitative research design was utilized to obtain data through focus group discussions and in-depth interviews with antenatal clinic attendees, health workers health facilities in the study area. Results. Awareness of PMTCT services and knowledge of its benefits was nearly universal, although socioeconomic, cultural and health system factors, including stigma and desire to prevent knowledge of serostatus, impede access to and utilization of services. Health system factors-lack of appropriate followup mechanisms, inadequate access to ARV drugs and poorly equipped manpower also contribute to low utilization of services. Conclusion. Reducing mother-to-child transmission of HIV in sub-Saharan Africa will be more effective when unique contextual factors are identified and addressed. Effectiveness of PMTCT interventions rests on a well functioning health system that recognize the importance of social, economic, cultural contexts that HIV positive pregnant women live in.
\end{abstract}

\section{Background}

In 2009, the United Nations AIDS Program (UNAIDS) reported that 430,000 of the approximately 2.5 million children under the age of 15 living with HIV were newly infected, the majority in sub-Saharan Africa [1]. Many of these children acquired the infection from their mothers during pregnancy, birth, or breastfeeding. Timely administration of antiretroviral drugs to a HIV-positive pregnant woman and her newborn child significantly reduces the risk of mother-to-child transmission [2]. Now recognized as an attainable public health strategy, preventing mother-to-child transmission (PMTCT) has four basic components: (i) prevention of primary infection among women, (ii) prevention of unintended pregnancies among HIV positive women, (iii) provision of specific interventions to reduce the risk of mother-to-child transmission, and (iv) provision of care, treatment and support to HIV infected women, their infants and families $[3,4]$. Providing highly active antiretroviral therapy to a woman will reduce viral replication and viral load during pregnancy, and as a postexposure prophylaxis, prevent infection in newborns $[3,5,6]$.

Interventions to reduce pediatric HIV infection have become readily available worldwide, especially in low and middle-income countries. In 2009, 53\% of HIV-infected pregnant women worldwide received antiretroviral (ARV) drugs to prevent mother-to-child transmission [7]. While coverage is increasing in sub-Saharan Africa, ranging from $8 \%$ in some settings to $54 \%$ in others [3], PMTCT programs in the continent are still plagued by multiple problems. For instance, many HIV positive pregnant women still face constraints in accessing ARV drugs because they refuse to participate or are lost to followup in existing programs. Health system factors (critical shortage of personnel, lack 
of skilled attendant at birth, poor infrastructure, and inadequate supply of PMTCT kits) as well as individual and sociocultural factors (stigma, nonawareness of PMTCT services, lack of spousal and family support, loss to followup, negative experiences with hospital staff, the preference for home delivery) have been highlighted as barriers in the literature [8-13].

Ethiopia, like most countries in sub-Saharan Africa, is experiencing a high prevalence of HIV with about $2.1 \%$ of the adult population living with the virus $[14,15]$ largely due to heterosexual transmission. Besides heterosexual transmission, vertical transmission of HIV from mother-to-child accounts for more than $90 \%$ of pediatric AIDS [16]. To reduce the number of mother-to-child HIV infections, the government set a goal of universal access and increased the capacity for the delivery of HIV counseling and testing, prevention of mother-to-child transmission, and provision of ARVs by about 2-, 6-, and 8-fold, respectively [15]. Despite this increase, the number of women accessing these services is still low. For instance, after more than 5 years since the goal for universal access was established, less than 7,000 HIV positive pregnant women received ARV prophylaxis, representing about $19 \%$ of the annual targets [16]. Similarly, Nigatu and Woldegebriel [17] showed from nationally available data that $47 \%$ of known HIV positive pregnant women were not receiving ARVs and $62 \%$ of known HIV exposed infants were not receiving ARVs, alarming results that are consistent with findings from other low-income countries $[10,11,13,15,18,19]$.

The reasons why HIV positive pregnant women are not accessing PMTCT services or return for followup varies from one context to another. Generally however, biological, operational, and local-contextual factors continue to impede the uptake of PMTCT services in many parts of sub-Saharan Africa even when services are available $[3,13]$. To address the problem of low utilization of PMTCT interventions, it is important to highlight and understand unique contextual factors and how they affect the performance of PMTCT programs. Few studies exploring these issues in Ethiopia have used quantitative or mixed method approaches [17, 19-22] and none to our knowledge has qualitatively explored these factors in Arba Minch to allow for rich and detailed description of peculiar contextual factors that facilitate or inhibit the effectiveness of PMTCT interventions. The aim of this study was to highlight and describe specific sociocultural, health system and operational barriers that contribute to the low uptake of PMTCT services in Arba-Minch, Ethiopia. Understanding the factors that affect the success of PMTCT, programs will contribute valuable information that will aid in developing new strategies and policies towards achieving the targets of universal access not only in Arba Minch and elsewhere in Ethiopia, but in similar communities across sub-Saharan Africa.

\section{Data and Methods}

2.1. Study Setting. The study setting is Arba Minch, the largest town in the Gamo Gofa zone located in western side of the Great Rift Valley in the Southern Nations, Nationalities and People's Region of Southern Ethiopia.
In addition to the main town, there are several villages inhabited by people who rely primarily on farming as a source of livelihood. Arba Minch is the administrative headquarters of Gamo-Gofa zone and boasts an array of economic and educational institutions, including the Arba Minch University. It is also a major tourist center situated about 500 kilometers from the capital, Addis-Ababa and 275 kilometers from the regional town of Awassa. Arba Minch was chosen as the study setting due to reports of increasing in HIV prevalence [23] and subsequent large number of HIV positive pregnant women needing antenatal care and access to PMTCT services. Of the 63 PMTCT sites located in the Southern Nations, Nationalities and People's region of Ethiopia in 2008, only 4 were sited in the Gamo Gofa zone to serve an estimated population of nearly half a million residents. Two major health facilities-the district hospital and the health centre were included in the study. Provision of antiretroviral therapy in Arba Minch Hospital commenced in 2003 and continued with the introduction of national free antiretroviral program launched by the government in 2005.

\subsection{Research Design and Data Collection. The study was} based on an exploratory qualitative research design to obtain data through focus group discussions and in-depth interviews. Interviews and discussion groups were held in two health facilities, purposively selected for the study. Both are equipped with basic facilities for the provision of HIVand AIDS-related services including voluntary counseling and testing, prevention of mother-to-child transmission and provision of highly active antiretroviral therapy. Although the district hospital is equipped to cater for a population of 250,000 , it now serves an estimated population of nearly 500,000 , while the health centre, originally equipped to cater for not more than 25,000, now caters for far more due to a continuously growing population in the area.

Qualitative data consisted of focus group discussions and in-depth interviews that were held with clinic attendees, health facility staff, and other key informants. In each facility, four focus group discussion sessions, consisting of 8 or 10 participants were held, equally divided between clinic attendees and health facility staff. Participants were recruited on the basis of homogeneity, convenience, and willingness to participate. Health workers (nurses, HIV counselors, midwives, etc.) in the facilities were recruited if they were affiliated with any of the following units: antenatal care, labor and delivery, voluntary counseling and testing, and prevention of mother-to-child transmission of HIV. One discussion group with health workers was held in the morning and the other in the evening in order to accommodate participants' availability. Antenatal clinic attendees were recruited after they were approached, briefed about the purpose of the research and consented to participate in focus groups interviews. In-depth interviews were conducted with one matron/head nurse, one physician at the district hospital, and 2 male and 2 female community leaders. All discussions and interviews were conducted in Amharic, the local language, by a team of three, including a moderator, note taker, and an observer. 
2.3. Data Analysis. We used Nvivo version 9 for qualitative data analysis, which followed a thematic framework. First, tapes from focus groups and interviews were transcribed and translated from Amharic to English before they were double checked for consistency and accuracy by the authors and other independent native speakers of Amharic. Where possible, transcripts were sent back to each interviewee for validation and to ensure accuracy in the data generated before analysis. Following this, the authors independently reviewed the transcripts several times to become familiar with the content before the process of sorting, coding, and theme identification $[24,25]$.

Following data validation, themes were developed based on an inductive and deductive process of issues that emerged from the interviews [25-27]. Each author initially developed themes independently, which were subsequently compared before deciding on which themes to report on. Furthermore, index cards were used to develop codes that were then systematically applied to the themes. This process ensured the mapping of themes and facilitated the application of relevant codes and themes. Following this process, a final list of codes and themes was derived and applied to the data. In presenting the data, relevant verbatim quotes are reported to aid in the interpretation of the data.

\section{Results}

3.1. Respondents' Characteristics. There were seventy-two participants in the focus group discussions. The health workers included antenatal care providers, pediatric nurses, midwives, auxiliary nurses, community health workers, and HIV counselors. The majority were women, have a mean age of 32.4 years and 5.6 years of work experience. Antenatal clinic attendees were all married and had a mean age of 23.5 years. About two-thirds were residents of the main town and the rest from surrounding villages. The majority was either Protestant or Orthodox Christian, reflecting the dominant religious groups in the study area. Although more than two-thirds of the women, the majority reported low incomes given that the majority was not in formal or selfemployment. The reported average household income was 727 Ethiopian Birr (ETB) (42.5USD) with the highest being 7168 ETB. In-depth interviewees were 3 women and 3 men including a matron/head nurse, one doctor, and 2 female and 2 male community leaders with ages ranging from 40 to 65 years.

3.2. Awareness of and Availability of PMTCT Services. We examined participants' awareness and knowledge of PMTCT and availability of related services. Awareness of PMTCT services in health facilities was universal with all respondents indicating they knew that services were available. The majority also indicated that testing for HIV was important for pregnant women since "there is a possibility of transmission from mother-to-child". They further indicated that antenatal visits, counseling, hospital delivery, use of ARV drugs, and avoiding breastfeeding were measures that could prevent HIV transmission to an unborn child.
3.3. Barriers to Utilization of PMTCT Services. We reviewed antenatal service statistics from the two facilities to determine the number of women returning to the facilities to deliver their babies after visiting the clinics and diagnosed with HIV. Of the 74 women diagnosed with HIV in the 3 months prior to this study, only $5(7 \%)$ returned to the facilities to deliver their babies. Twenty-nine HIV positive pregnant women who were not enrolled on the ANC-PMTCT register were also recorded to give birth in both facilities.

Interviews with focus group participants and key informants revealed reasons why many women do not return to health facilities to give birth. Socioeconomic, cultural, and health system factors compel HIV positive pregnant women to stay away from health facilities when they are due to give birth. Focus group participants report that HIV related stigma could be inhibiting the effectiveness of PMTCT services in Arba Minch as women who test positive want to keep such information from close relatives and significant others who play an active role during child birth. Culturally, husbands or older female relatives tend to be present during labor and/or delivery, and for HIV positive women who desire to keep their status confidential, the tendency is often to avoid returning to facilities where they were diagnosed, preferring a home or assisted delivery in a facility where their status is unknown. As one FGD participant reported:

"It is a shameful thing to be diagnosed with HIV. How would you say you got it? Even if your husband is responsible, the people will conclude it is your fault. To avoid trouble, most women don't give birth in the hospital where their status is known so that relatives will not become suspicious when they give special medication [ART] to the mother and baby shortly after birth" (Clinic Attendee, FGD participant)

"It [HIV] is a big issue in this community, so people do whatever is necessary to prevent others from knowing their status because of stigma. That is why women who are positive do not go back to where they are diagnosed for fear of meeting someone who will know them and tell their family" (Clinic Attendee, FGD participant)

"...The rules clearly state that we must initiate follow up and aggressively so, but unfortunately, many HIV positive women run away from giving birth in the hospital because they don't want anyone to know they are HIV positive, especially their relatives who will accompany them into the labor room" (Nurse Midwife, FGD participant)

\footnotetext{
"Some women are very cooperative when it comes to testing for HIV and being put on ART. However, there are problems when we expect them to return to the facility to give birth. Several things are responsible for this problem including the cultural practices, the nature of the women's work, low socioeconomic status and frequent change of their addresses, which makes follow up very tedious" (PMTCT Nurse, FGD participant)
} 
Stigmatizing attitudes also manifest within the hospital setting. For instance, participants in focus groups suggested that many health workers are not enthusiastic about handling deliveries for women who are known to be HIV positive for fear of accidental infection. One key informant reported "many health workers don't have the necessary skill and equipment to confidently handle delivery for an HIV positive woman and given the risk of accidental exposure, most nurses shy away from dealing with such patients." The concern about health workers' ability to handle HIV positive patients was repeated in several group and individual interviews, thus underscoring the need for the training of health facility staff in safety precautions and procedures. As another key informant puts it:

"Those of us who work here still need a lot of training to effectively handle the challenges of PMTCT beyond counseling women, placing them on the PMTCT register and encouraging them to return to the hospital" (Health facility Key Informant).

These problems are compounded by the fact that other health system factors also contribute to the low numbers of HIV positive women who return to health facilities to give birth. A key issue identified in interviews is inadequate monitoring and referral structures within the health system. For instance, as one matron reported "we don't have appropriate monitoring strategies to follow up when HIV positive women miss antenatal appointments. The fact that we continue to see previously undiagnosed HIV positive women during labor indicates to me that something is not right". Another key informant reported:

\begin{abstract}
"Most women are still able to avoid the system since we have no way to trace them so that they don't go to other facilities when the time comes for delivery... health facilities should be able to share information about HIV positive women so that our efforts to prevent the fetus from becoming infected can be more effective. It is not just enough to give counseling and ART, we need strategies to address current lapses within the health system" (Health facility Key Informant)
\end{abstract}

Inadequate human resource capacity was also identified as a constraint to effective delivery of PMTCT services in the study areas. Key informants and health workers who participated in focus group discussions reported that PMTCT units are inadequately staffed and available ones are overworked. Thus, as one health facility key informant reported: "the problem is not just that of training, but we need more staff to increase our capacity to provide effective services".

Other factors that constrain the effectiveness of PMTCT services include inadequate ARV supply, cost of accessing services and low-socioeconomic status of women. Focus group participants and key informants reported that access to PMTCT services is severely restricted for many lowincome women who live on the outskirts and have to travel long distances to reach health facilities.
"Several times, we exhaust our supplies of ARTs for both the mothers and children and this hinders our ability to intervene in those critical early hours of the life of a new born during which first line drugs should be administered. I don't blame the women if they fail to return because they lack confidence in our ability to prevent their child from getting infected" (Health facility Key informant).

"The women who come here are poor and many find it difficult to pay the fees before they are treated. I am not surprised many don't show up ... when they cannot afford to pay for transport or drugs, they will be discouraged" (Health worker FGD participant).

\section{Discussion}

There are nearly 1.1 million Ethiopians living with HIV and prevalence continues to increase despite efforts by the Ethiopian Ministry of Health and the US government through United States Aid Agency (USAID) initiatives. Despite these efforts, only $8 \%$ of eligible HIV positive pregnant women are currently receiving antiretroviral drugs to prevent motherto-child transmission. Although PMTCT interventions can be highly successful in reducing the risk of HIV transmission, access to and utilization of PMTCT services is still limited in many parts of sub-Saharan Africa owing to a variety of structural-contextual factors. Although Ethiopia has made progress in the quality of PMTCT services provided, coverage, especially in rural areas, still remains abysmally low. In Arba Minch, our findings highlight individual, sociocultural, and health system related factors that contribute to the low usage of PMTCT among HIV-positive women.

Overall, study participants demonstrated high levels of awareness and knowledge regarding the importance of using PMTCT services to prevent maternal transmission of HIV to their children. While women recognize the importance of preventing mother-to-child transmission and have all received HIV counseling and testing (HCT), their desperation to conceal their positive status from close family members compels them to stay away from the facilities where they were diagnosed and to give birth either at home or in facilities where their HIV-positive status is not known. As women are concerned about the ramifications of close family members becoming cognizant of their positive status, efforts to improve the effectiveness of PMTCT programs must identify and involve these significant others in the process to help dissipate the stigmatization of HIV-positive women. The importance of family support for women living with HIV can never be overestimated especially when husbands and mothers-in-law in particular have traditionally played key roles in the care and maintenance of expectant mothers. As studies have highlighted, there exists huge potential for improving the effectiveness of PMTCT programs when people living with HIV have the support of their families $[13,17,28-33]$. 
Stigma is still a major feature of the AIDS milieu in many communities and people living with HIV experience stigma at various levels, including health workers [34-36]. Subtle stigmatizing attitudes and discriminatory behavior from health workers, which are often overlooked, can limit the potential impact of interventions for people living with HIV. For example, health workers' reluctance or refusal to assist HIV positive women during the birthing process due to their concerns of contracting HIV through occupational exposure may be the result of poor training or lack of personal protective equipment. Although such concerns are not unfounded, failure to address them allows the perpetuation of stigma and discrimination against women and leads to a less desirable enrollment in prevention, care, and treatment programs for people living with HIV [13]. Providing mandatory education that focuses on changing and addressing attitudes and how to share sensitive information about people living with HIV to health workers will be an important step in changing subtle, but powerful stigmatizing attitudes among health workers.

Lack of knowledge of a woman's HIV status and/or availability of skilled birth attendance represents a missed opportunity for initiating prophylactic ARV therapy among pregnant women and for postexposure prophylactic ARV therapy for newborns. As previous studies have shown, women's struggle with serostatus disclosure and stigma makes it difficult for them to participate in PMTCT programs and to initiate and continue the use of ARV therapy for their own health and that of their children [10, 13, 37]. Since 2006, antenatal clinics in Ethiopia routinely offer HIV testing to all pregnant women (opt-out approach) [14], with the aim of getting as many women as possible to be tested for HIV infection. In countries that implement this approach, there is evidence suggesting wider coverage in HIV testing but a low enrollment in PMTCT services as women try to conceal their status in communities where stigma is still pervasive $[10,18,38,39]$. This can contribute to an increase in the number of HIV positive women not linked to care and failure to prevent mother-to-child transmission.

Structural inadequacies also hinder the effectiveness of PMTCT services in many low- and middle-income countries. Health system factors, including the critical shortage of skilled manpower, high staff turnover, inadequate physical infrastructure, poor quality of pre-/posttest counseling, lack of monitoring and referral programs, inadequate supply of antiretroviral drugs, and poor quality of service delivery have all been highlighted as barriers to the effectiveness of PMTCT services $[17,20,40]$. Our findings confirm that similar factors play a role in the lack of effectiveness of PMTCT services in Arba Minch, for example, how the organization of pre- and posttest counseling can compromise confidentiality of results. In addition, lack of appropriate monitoring and referral systems for HIV positive women meant there was no way to trace those who are lost to follow up even if they go to other facilities to give birth.

Failure to utilize PMTCT services was further complicated by cost of accessing services. Low incomes meant many women could not afford the huge cost associated with transportation and other logistics of accessing PMTCT services that were offered for free. This is even more challenging for rural residents who find transportation cost to the clinics prohibitively expensive due to the long distances that must be travelled. The implication is that even when women recognize the need to and want to access care, the inability to overcome the transportation barrier constitutes another challenge.

To address these problems, it is necessary to establish guidelines and protocols that regulate the conduct of health workers caring for HIV positive pregnant women. Additionally, it is important to develop monitoring and referral structures to promote followup and complement already existing PMTCT services. This will require investments in cost effective administrative and information sharing infrastructure at local, regional, and national levels, while taking into account the need to promote patients' rights to confidentiality and privacy. While the opt-out strategy has contributed to an increase in the number of pregnant women testing for HIV, its potential to limit the proportion that is linked to care, given deep-rooted stigma, highlights the need to consider peculiar contextual factors that may limit the effectiveness of this approach. Moreover, the decentralization of the response to HIV/AIDS as demonstrated in the Millennium AIDS Campaign launched in 2006 to improve access to treatment is a strategy that should be vigorously implemented, while addressing the sociocultural and other contextual factors that have been highlighted in this study.

To improve the effectiveness of PMTCT programs in Arba Minch and similar contexts, it is important to change perceptions of HIV that encourage stigma and discrimination against PLHIV at both local and national levels. It will help to emphasize that with access to ARV therapy and proper treatment and care, people living with HIV and AIDS can lead healthy, productive, and long lives. Furthermore, interventions to mitigate the impact of HIV should involve all stakeholders including health workers, families, and communities. For example, the use of health extension workers (HEWs) has contributed to improving health outcomes [4143] in remote rural areas, thus demonstrating that such strategies can catalyze a change in how people perceive and treat people living with HIV.

\section{Conclusion}

This study has a number of limitations including the purposively selected facility-based sample of HIV positive pregnant women from one district of Ethiopia, which may not adequately reflect the circumstances of HIV positive pregnant women who do not frequent health facilities. In addition, our data may be inhibited by reporting bias due to interviews being conducted within health facilities and participants' fear of the negative repercussions that may result from them reporting their opinions. Furthermore, the use of ANC service statistics may have included women with different characteristics to those not accessing PMTCT services.

Despite these limitations, the policy implication of the study is clear-the effectiveness of PMTCT programs in low- and middle-income countries rests not only on a well functioning health system but also on the interplay between social, cultural and peculiar contextual circumstances. Our 
findings, which are consistent with those of similar studies in other parts of Ethiopia [17, 20,40] show that overall, the social and cultural issues highlighted in this study as well as the organizational and service delivery mechanisms (policies, procedures, and health system factors) through which PMTCT services are organized and delivered constitute serious challenges to the goal of universal access set by the government. To achieve the target in view of these findings will require a thorough review and reorganization of existing policies and current approaches to increase uptake in services among women, especially those living in areas similar to the study area. In addition, understanding how unique contextual factors limit women's access to or utilization of PMTCT services is critically important, if not crucial. Addressing these contextual factors requires engaging communities to understand the importance of PMTCT to develop innovative and sustainable strategies for removing the barriers that women face in accessing and utilizing PMTCT services.

\section{References}

[1] “United Nations AIDS Program (UNAIDS)," 2010, http:// www.unaids.org/globalreport/Global_report.htm.

[2] World Health Organization, Antiretroviral Drugs for Treating Pregnant Women and Preventing Infection in Infants: Towards Universal Access: Recommendations for a Public Health Approach, WHO, Geneva, Switzerland, 2006.

[3] World Health Organization, "Prevention of Mother to Child Transmission (PMTCT). Briefing note. Department of HIV/ AIDS," 2007, http://www.who.int/hiv/pub/toolkits/PMTCT\%20HIV\%20Dept\%20brief\%20Oct\%2007.pdf.

[4] World Health Organization, "New guidance on prevention of mother-to-child transmission of HIV and infant feeding in the context of HIV," 2010, http://www.who.int/hiv/pub/mtct/ PMTCTfactsheet/en/index.html.

[5] N. Siegfried, L. van der Merwe, P. Brocklehurst, and T. T. Sint, "Antiretrovirals for reducing the risk of mother-to-child transmission of HIV infection," Cochrane Database of Systematic Reviews, no. 7, p. CD003510, 2011.

[6] F. Dabis, M. L. Newell, L. Fransen et al., "Prevention of mother-to-child transmission of HIV in developing countries: recommendations for practice," Health Policy and Planning, vol. 15 , no. 1 , pp. $34-42,2000$.

[7] World Health Organization, "Towards Universal Access Progress Report," 2010, http://www.who.int/hiv/pub/2010progressreport/en/.

[8] P. M. Barker, W. Mphatswe, and N. Rollins, "Antiretroviral drugs in the cupboard are not enough: the impact of health systems' performance on mother-to-child transmission of HIV," Journal of Acquired Immune Deficiency Syndromes, vol. 56, no. 2, pp. e45-e48, 2011.

[9] Z. P. Theilgaard, T. L. Katzenstein, M. G. Chiduo et al., "Addressing the fear and consequences of stigmatization-a necessary step towards making HAART accessible to women in Tanzania: a qualitative study," AIDS Research and Therapy, vol. 8, no. 1, p. 28, 2011.

[10] J. R. Chinkonde, J. Sundby, and F. Martinson, "The prevention of mother-to-child HIV transmission programme in Lilongwe, Malawi: why do so many women drop out," Reproductive Health Matters, vol. 17, no. 33, pp. 143-151, 2009.

[11] M. Manzi, R. Zachariah, R. Teck et al., "High acceptability of voluntary counselling and HIV-testing but unacceptable loss to follow up in a prevention of mother-to-child HIV transmission programme in rural Malawi: scaling-up requires a different way of acting," Tropical Medicine and International Health, vol. 10, no. 12, pp. 1242-1250, 2005.

[12] World Health Organization, The World Health Report, Geneva, Switzerland, 2005.

[13] T. M. Painter, K. L. Diaby, D. M. Matia et al., "Women's reasons for not participating in follow up visits before starting short course antiretroviral prophylaxis for prevention of mother to child transmission of HIV: qualitative interview study," British Medical Journal, vol. 329, no. 7465, pp. 543-546, 2004.

[14] "Ministry of Health Ethiopia \& Federal HIV/AIDS Prevention and Control Office: Single Point HIV prevalence estimate," Addis Ababa, Ethiopia, 2007.

[15] "United Nations AIDS Program (UNAIDS)Epidemiological Fact Sheet on HIV and AIDS: Core Data on Epidemiology and Response. Ethiopia," 2008, http://apps.who.int/globalatlas/ predefinedReports/EFS2008/full/EFS2008_ET.pdf.

[16] "Federal HIV/AIDS Prevention and Control Office," In Multisectoral HIV/AIDS Response Annual Monitoring and Evaluation Report 202 EFY, 2011.

[17] T. Nigatu and Y. Woldegebriel, "Analysis of the prevention of mother-to-child transmission (PMTCT) service utilization in Ethiopia: 2006-2010," Reproductive Health, vol. 8, no. 1, article 6, 2011.

[18] T. Creek, R. Ntumy, L. Mazhani et al., "Factors associated with low early uptake of a national program to prevent mother to child transmission of HIV (PMTCT): results of a survey of mothers and providers, Botswana, 2003," AIDS and Behavior, vol. 13, no. 2, pp. 356-364, 2009.

[19] Z. Moges and A. Amberbir, "Factors associated with readiness to VCT service utilization among pregnant women attending antenatal clinics in Northwestern Ethiopia: a health belief model approach," Ethiopian Journal of Health Sciences, vol. 21, supplement 1, pp. 107-115, 2011.

[20] A. H. Mirkuzie, S. G. Hinderaker, and O. Mørkve, "Promising outcomes of a national programme for the prevention of mother-to-child HIV transmission in Addis Ababa: a retrospective study," BMC Health Services Research, vol. 10, article 267, 2010.

[21] O. Bolu, A. Anand, A. Swartzendruber et al., "Utility of antenatal HIV surveillance data to evaluate prevention of motherto-child HIV transmission programs in resource-limited settings," American Journal of Obstetrics and Gynecology, vol. 197, no. 3, supplement, pp. S17-S25, 2007.

[22] G. Worku and F. Enquselassie, "Factors determining acceptance of voluntary HIV counseling and testing among pregnant women attending antenatal clinic at army hospitals in Addis Ababa," Ethiopian Medical Journal, vol. 45, no. 1, pp. 18, 2007.

[23] D. Jerene, A. Endale, and B. Lindtjørn, "Acceptability of HIV counselling and testing among tuberculosis patients in south Ethiopia," BMC International Health and Human Rights, vol. 7, article 4, 2007.

[24] P. S. Maykut and S. Moorehouse, Beginning Qualitative Research: A Philosophic and Practical Guide, Falmer Press, London, UK, 1994.

[25] C. Pope and N. Mays, Qualitative Research in Health Care, Blackwell, 3rd edition, 2006.

[26] J. Green and N. Thorogood, Qualitative Methods for Health Research, Introducing Qualitative Methods, Sage, London, UK, 2004.

[27] D. Gray, Doing Research in the Real World, Sage, London, UK, 2009. 
[28] E. F. Falnes, K. M. Moland, T. Tylleskar, M. M. de Paoli, S. C. Leshabari, and I. M. S. Engebretsen, "The potential role of mother-in-law in prevention of mother-to-child transmission of HIV: a mixed methods study from the Kilimanjaro region, Northern Tanzania," BMC Public Health, vol. 11, article 551, 2011.

[29] R. Byamugisha, J. K. Tumwine, N. Semiyaga, and T. Tylleskär, "Determinants of male involvement in the prevention of mother-to-child transmission of HIV programme in Eastern Uganda: a cross-sectional survey," Reproductive Health, vol. 7, no. 1 , article 12, 2010.

[30] E. C. Larsson, A. Thorson, G. Pariyo et al., “Opt-out HIV testing during antenatal care: experiences of pregnant women in rural Uganda," Health Policy and Planning, vol. 27, no. 1, pp. 69-75, 2011.

[31] L. R. Østergaard and A. Bula, “"They call our children "Nevirapine babies?"': a qualitative study about exclusive breastfeeding among HIV positive mothers in Malawi," African Journal of Reproductive Health, vol. 14, no. 3, pp. 213-222, 2010.

[32] M. Conkling, E. L. Shutes, E. Karita et al., "Couples' voluntary counselling and testing and nevirapine use in antenatal clinics in two African capitals: a prospective cohort study," Journal of the International AIDS Society, vol. 13, p. 10, 2010.

[33] K. Peltzer, T. Mosala, O. Shisana, A. Nqueko, and N. Mngqundaniso, "Barriers to prevention of HIV transmission from mother to child (PMTCT) in a resource poor setting in the Eastern Cape, South Africa," African Journal of Reproductive Health, vol. 11, no. 1, pp. 57-66, 2007.

[34] B. Nattabi, J. Li, S. C. Thompson, C. G. Orach, and J. Earnest, "Factors associated with perceived stigma among peopleliving with hiv/aids in post-conflict northern Uganda," AIDS Education and Prevention, vol. 23, no. 3, pp. 193-205, 2011.

[35] R. Cianelli, L. Ferrer, K. F. Norr et al., "Stigma related to HIV among community health workers in Chile," Stigma Research and Action, vol. 1, no. 1, pp. 3-10, 2011.

[36] T. Whitaker, P. Ryan, and G. Cox, "Stigmatization among drug-using sex workers accessing support services in Dublin," Qualitative Health Research, vol. 21, no. 8, pp. 1086-1100, 2011.

[37] H. Muyinda, J. Seeley, H. Pickering, and T. Barton, "Social aspects of AIDS-related stigma in rural Uganda," Health and Place, vol. 3, no. 3, pp. 143-147, 1997.

[38] S. Walmsley, "Opt in or opt out: what is optimal for prenatal screening for HIV infection?" Canadian Medical Association Journal, vol. 168, no. 6, pp. 707-708, 2003.

[39] C. Sprague, M. F. Chersich, and V. Black, "Health system weaknesses constrain access to PMTCT and maternal HIV services in South Africa: a qualitative enquiry," AIDS Research and Therapy, vol. 8, no. 10, pp. 10-18, 2011.

[40] Federal Ministry of Health: Report on Situational Analysis of the National PMTCT of HIV/AIDS Program Response in the Broader RH/MCH Context in Ethiopia, Addis- Ababa, 2009.

[41] H. Banteyerga, "Ethiopia's Health Extension Program: improving health through community involvement," MEDICC Review, vol. 13, no. 3, pp. 46-49, 2011.

[42] K. Hermann, W. Van Damme, G. W. Pariyo et al., "Community health workers for ART in sub-Saharan Africa: learning from experience-capitalizing on new opportunities," Human Resources for Health, vol. 7, article 31, 2009.

[43] W. Wakabi, "Extension workers drive Ethiopia's primary health care," The Lancet, vol. 372, no. 9642, p. 880, 2008. 


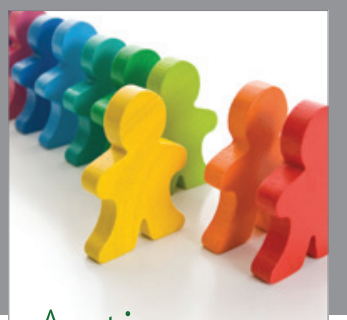

Autism

Research and Treatment
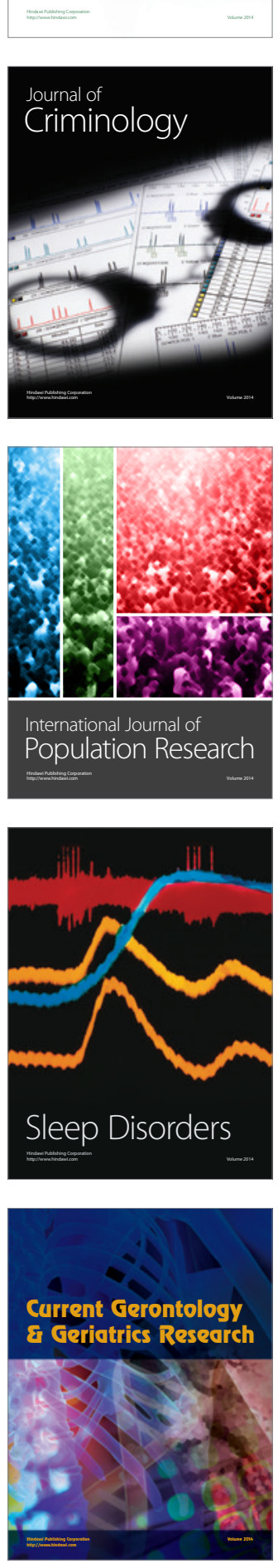
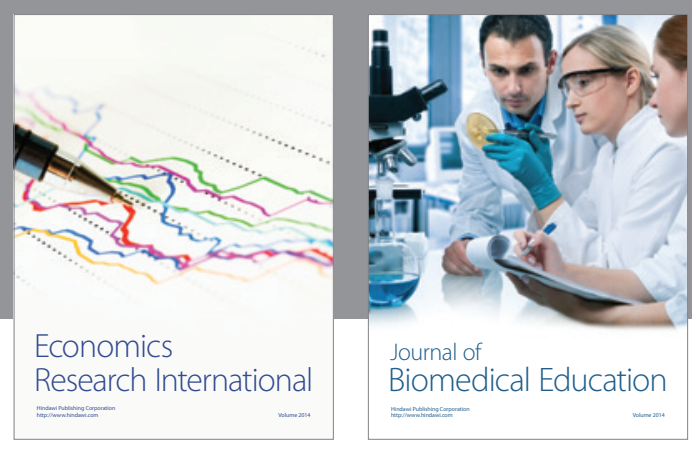

Journal of

Biomedical Education

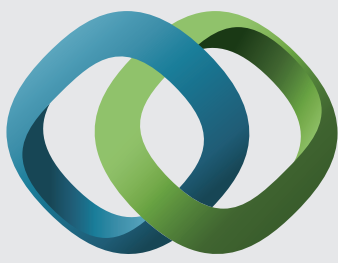

\section{Hindawi}

Submit your manuscripts at

http://www.hindawi.com
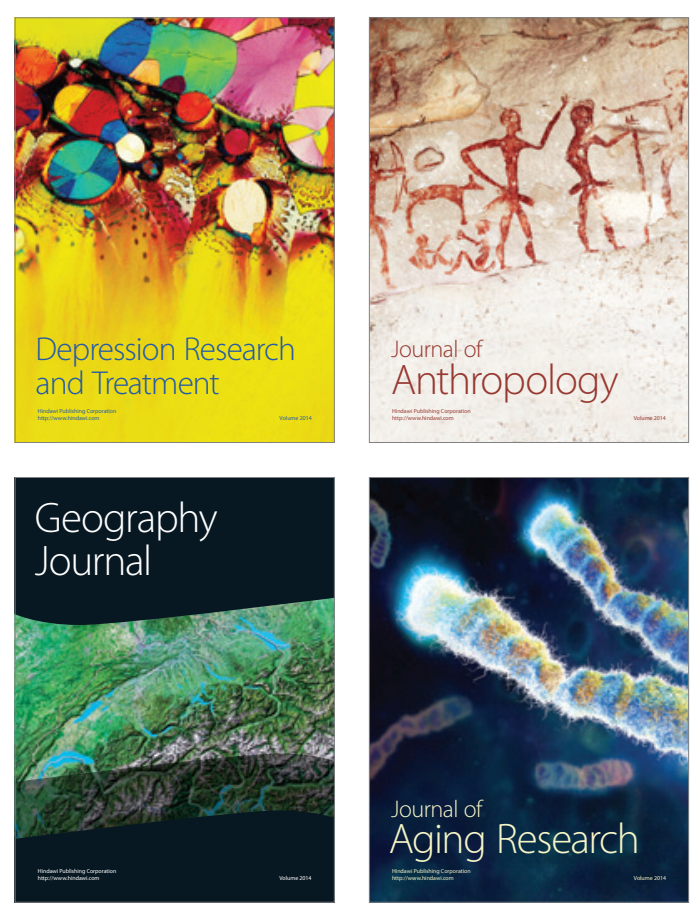

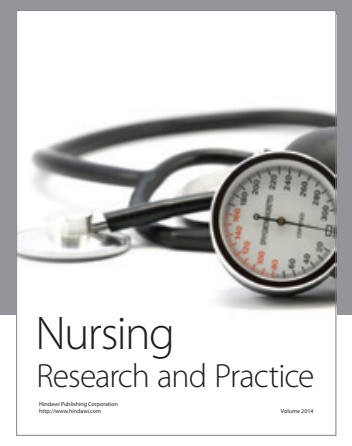

Nursing

Research and Practice

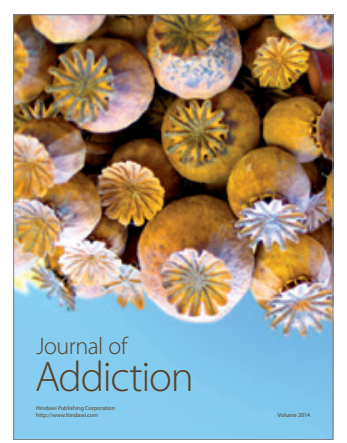

Child Development

Research

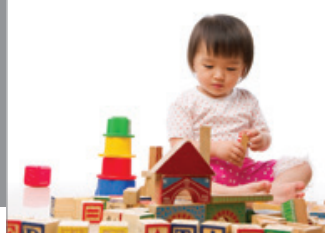

迥
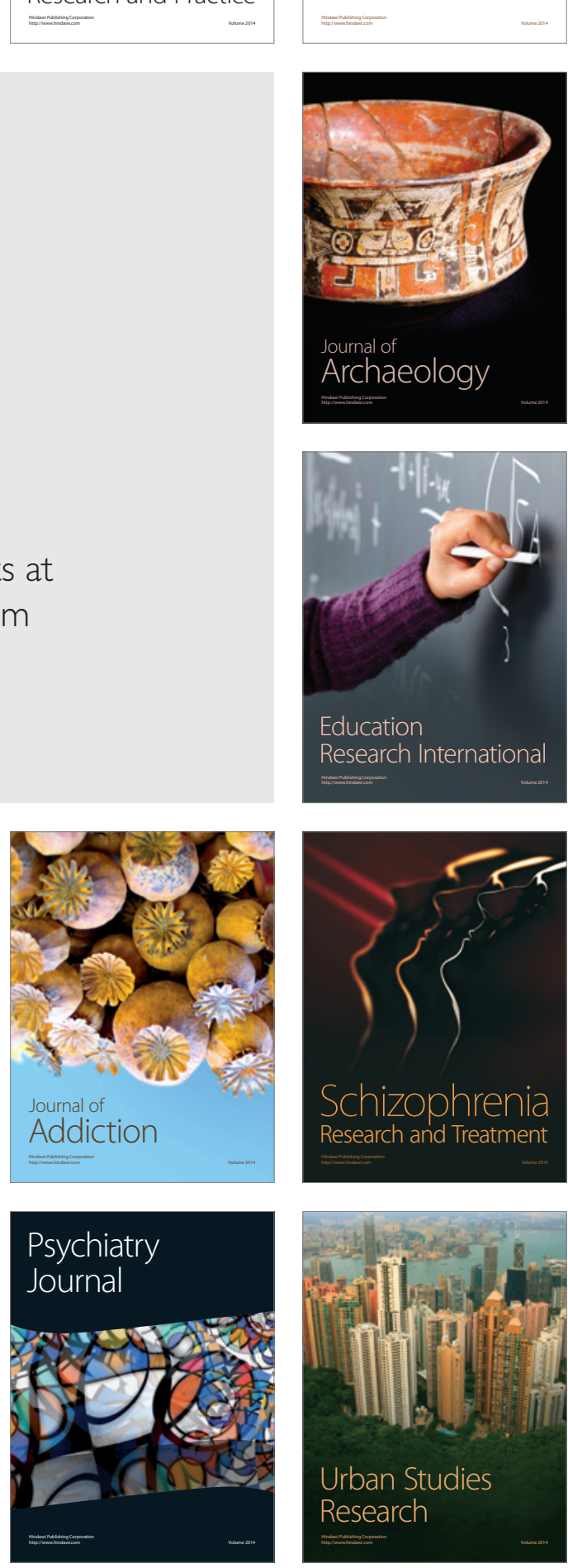